\title{
Critical fields and fluctuations determined from specific heat and magnetoresistance in the same nanogram $\operatorname{SmFeAs}(\mathrm{O}, \mathrm{F})$ single crystal
}

\author{
S.Galeski*, P.W.J. Moll, N.Zhigadlo, K.Mattenberger, B. Batlogg \\ Solid State Physics Laboratory, ETH Zurich, CH-8093 Zurich, Switzerland ${ }^{*}$
}

\begin{abstract}
Through a direct comparison of specific heat and magneto-resistance we critically asses the nature of superconducting fluctuations in the same nano-gram crystal of $\operatorname{SmFeAs}(\mathrm{O}, \mathrm{F})$. We show that although the superconducting fluctuation contribution to conductivity scales well within the 2DLLL scheme its predictions contrast the inherently 3D nature of $\operatorname{SmFeAs}(\mathrm{O}, \mathrm{F})$ in the vicinity $T_{c}$. Furthermore the transition seen in specific heat cannot be satisfactory described either by the LLL or the XY scaling. Additionally we have validated, through comparing $\mathrm{H}_{\mathrm{c} 2}$ values obtained from the entropy conservation construction $\left(\mathrm{H}_{\mathrm{c} 2 \mathrm{ab}}=-19.5 \mathrm{~T} / \mathrm{K}\right.$ and $\left.\mathrm{H}_{\mathrm{c} 2 \mathrm{ab}}=-2.9 \mathrm{~T} / \mathrm{K}\right)$, the analysis of fluctuation contribution to conductivity as a reasonable method for estimating the $H_{c 2}$ slope.
\end{abstract}

\section{INTRODUCTION}

The surprising discovery of superconductivity at $26 \mathrm{~K}$ in $\mathrm{LaFeAsO}$ in 2008 was a beginning of a new era for superconductivity 1 . Soon after the initial discovery a great effort was taken to reach higher transition temperatures, resulting in a discovery of dozens of iron based superconductors with new compounds still being synthesized $^{2}$. However promising, the abundance of new structures was not followed by the availability of high quality macroscopic samples. In particular crystals of the '1111' family, with the highest $T_{c}=55 \mathrm{~K}$, usually grow as flakes of $100-200 \mu \mathrm{m}$ diameter, ${ }^{3}$ making it challenging to study their bulk thermodynamics. In the face of such difficulties newly discovered superconductors are traditionally characterized by their transport properties. However the influence of possible filamentary and surface superconductivity together with the defect-induced vortex pinning the resistive transition tends to be difficult to interpret.

This is particularly visible when determining the $H_{c 2}$ slope from the typically smooth and featureless resistive data - depending on the chosen criterion for the transition temperature $(10,5090 \%$ of normal state resistivity) one might obtain $H_{c 2}$ slopes differing by more than a factor of $2-33^{4}$.

One debate originating from these issues is the discussion of dimensionality of the superconducting fluctuations in $\operatorname{SmFeAs}(\mathrm{O}, \mathrm{F})$ with reports of $2 \mathrm{D}$ and 3D behaviour ${ }^{[5] 6}$. In the first work Palecchi et al. reported that the superconducting fluctuation contribution to conductivity could be well parametrized within the 2D-LLL scaling scheme in stark contrast to the second study of Welp et al. who suggested the prevalence of 3D-LLL scaling of the superconducting contribution to specific heat in fields up to 8T. One possible explanation of this apparent discrepancy could be the sample variability. On the other hand the analysis of scaling of the superconducting fluctuation contribution to conductivity suffered from the lack of high quality single crystals and was performed on a polycrystalline sample making a proper analysis of fluctuation conductivity very difficult.

Here we measure on the same single crystal of Sm-
$\mathrm{FeAs}(\mathrm{O}, \mathrm{F})$, both heat capacity and resistivity near the superconducting transition in fields up to $14 \mathrm{~T}$ applied parallel and perpendicular to the FeAs layers. Analysis of the phenomenology of the resistive transition shows that the low temperature part of the transition is strongly influenced by the vortex dynamics. On the other hand, the onset of the transition can be well accounted for as originating from fluctuation conductivity (with the same $\mathrm{H}_{\mathrm{c} 2}$ slope as found in specific heat ) and following the scaling form of the 2D Lowest Landau Level (LLL) theory. The appearance of the specific heat anomaly accompanying the transition also reveals a significant presence of fluctuations, however they cannot be well described neither within the LLL nor the XY scaling schemes.

Our analysis shows that despite many similarities with the cuprates the multi-band nature of the iron pnictides makes them even more complex.

\section{EXPERIMENTAL}

$\operatorname{SmFeAs}(\mathrm{O}, \mathrm{F})$ single crystals were grown under high pressure in a $\mathrm{NaCl} / \mathrm{KCl}$ flux, typically they grew in the form of $5-10 \mu m$ thick platelets with $100-200 \mu m$ diameter ${ }^{3}$. In the course of this study we have used two crystals of approximately $40 \times 50 \times 5 \mu \mathrm{m}^{3}$ and $50 \times$ $100 \times 10 \mu \mathrm{m}^{3}$. The size of the crystals was estimated from electron microscope images. In order to perform specific heat measurements on such small samples we have employed membrane nano calorimeters ${ }^{7}$ combined with the 345 method allowing us to measure specific heat of samples as small as $30 \mathrm{um}$ in diameter (corresponding to $\sim 50 \mathrm{ng} \sqrt{8}$. After the specific heat measurements the sample was transferred onto a silicon wafer and subsequently trimmed into a Hall bar and electrically connected using the focused ion beam, as shown in the inset of Fig 3110 . Measurements were performed in a Quantum Design PPMS cryostat equipped in a 14 Tesla magnet. 

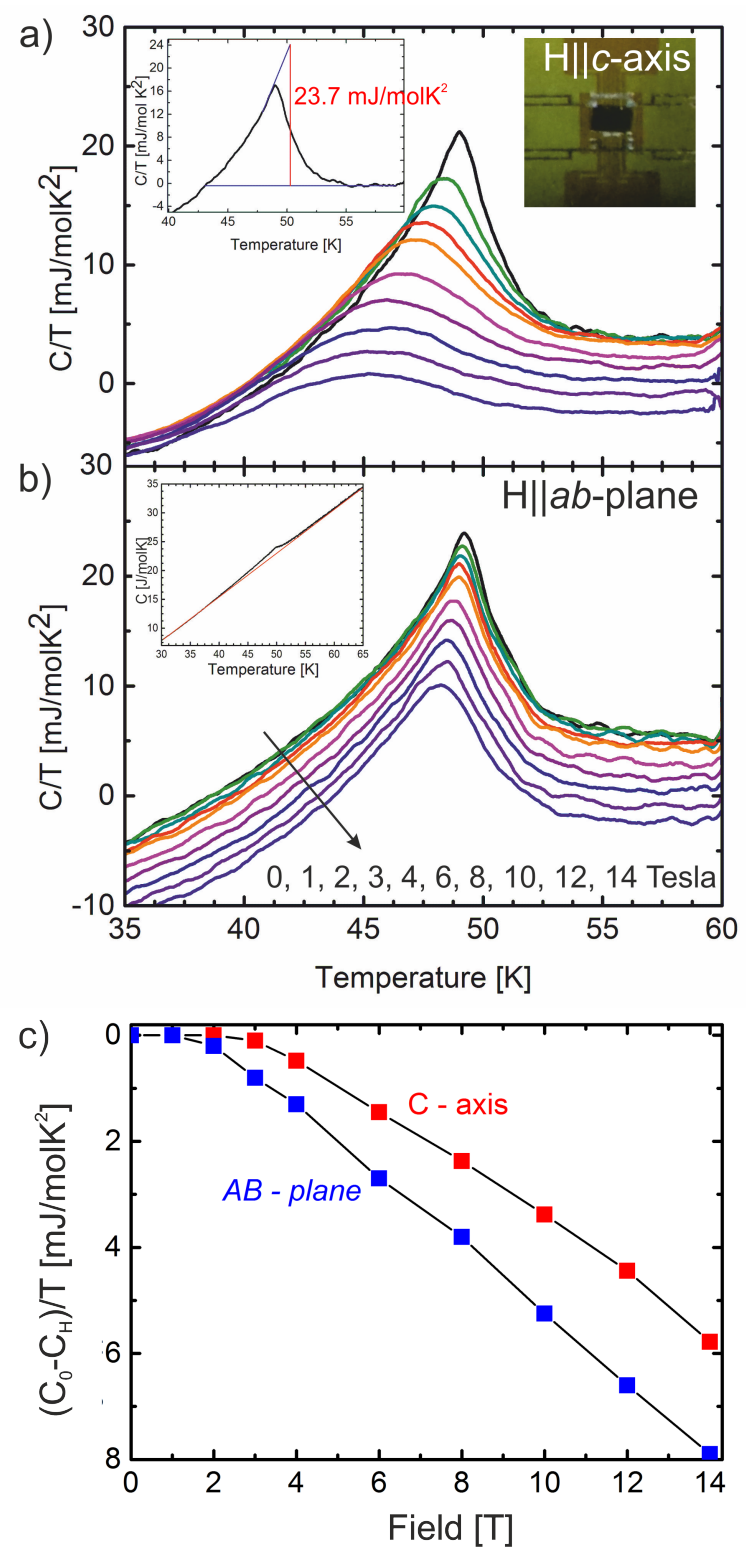

FIG. 1. Variation of the specific heat anomaly of single crystal $\mathrm{SmFeAs}(\mathrm{O}, \mathrm{F})$ with the magnetic fields applied along the $c$ axis (upper panel) and parallel to the $a b$-plane (middle panel). The inset left of the upper panel exemplifies the procedure used for extracting $T_{c}$ at various fields. The inset of the middle panel presents the total measured specific heat. The bottom panel shows the field dependence of the difference $C(0 T)-C(H)$.

\section{SPECIFIC HEAT}

Reliable measurements of specific heat of the '1111' family or in fact of any crystals that are not of macroscopic size seem to be a formidable task and there are only a few reports of thermodynamic bulk measurements performed on nanogram samples6/811.

In order to perform these experiments we have employed membrane based nano-calorimeters manufactured by Xensor Integration. These chips although designed for operation in temperatures up to $700 K$ turned out to work very reliably at low temperatures down to $1.8 \mathrm{~K}$. Unlike most nano-calorimeters used in condensed matter physics thermometry in these devices does not relay on resistive thermometers but is based on a set of 6 compensated silicon thermopiles. Such a design proves to be especially useful when employing the 345 method as it allows a direct measurement of the temperature difference between the sample and the chip frame (For design details please refer to Xensor technical note ${ }^{7}$ ).

The superconducting specific heat anomaly amounts to less than $5 \%$ of the total specific heat (inset of Fig $1 \mathrm{~b}$ ). To extract meaningful thermodynamic information regarding the superconducting transition we follow the procedure introduced by Welp. et al! ${ }^{[12}$ and subtract a linear background. However for clarity we have subtracted the same zero field background line from all curves, explicitly showing the field dependence of the normal state specific heat. (Figure 1, bottom panel).

Interestingly the normal state specific heat above the transition is reduced in a magnetic field (Fig,1 $)$. This can be tentatively ascribed to the modification of the crystal-field split energy levels of the Sm $4 f$ electrons, as no such suppression in observed in the $\mathrm{Nd}$ - based counterpart 13 .

The specific heat near $T_{c}$ is shown in Fig, 1 . We have fitted a 'mean field jump' (inset of Fig 1a) to the transitions assuming entropy conservation as exemplified in the inset of Fig, 1, yielding the following parameters: $T_{c}=50.5 \mathrm{~K}$, the upper critical field slopes $H_{c 2}^{\prime}$, Fig. 1 with the field parallel to the $c$-axis: $\sim 2.9 \mathrm{~T} / \mathrm{K}$ and $\sim 19.5 T / K$ parallel to the $a b$-plane. The estimate of the jump hight yielded $\Delta C / T=17.7 \mathrm{~mJ} / \mathrm{mol} \mathrm{K}^{2}$ for crystal I and $23.7 \mathrm{~mJ} / \mathrm{mol} \mathrm{K}^{2}$ for crystal II. These values are in fair agreement with data previously reported by Welp et al. who estimated the anisotropy parameter $\Gamma=\frac{H_{c 2}^{a b}}{H_{c 2}^{c}}=8$ with the critical field slope along the $c$-axis as $-3.5 \mathrm{~T} / \mathrm{K}$.

A qualitative investigation of the shape of the specific heat anomalies reveals a strong superconducting fluctuation contribution with the high temperature fluctuation tail extending almost $5 \mathrm{~K}$ above bulk $T_{c}$, suggesting some similarities to the cuprate superconductors. In the case of the cuprates two scenarios were proposed to describe the behaviour of specific heat in the vicinity of $T_{c}$ in magnetic fields: the 2D and 3D Lowest Landau Level (LLL) theory and the 3D XY model 14 .

It was shown that the LLL theory should be a valid approximation for describing superconducting fluctuations as soon as the magnetic field becomes strong enough to confine the order parameter to the lowest Landau level, what translates to a criterion $H>H_{L L L}$ with $H_{L L L} \approx G_{i} H_{c 2}(0)$. The LLL theory predicts that specific heat in the vicinity of $H_{c 2}$ should be well described, depending on dimensionality of the fluctuations by 14 . 


$$
\begin{aligned}
& \frac{d C}{d T} H^{1 / 2}=F_{2 D}^{C}\left(\frac{T-T_{c}(H)}{(T H)^{1 / 2}}\right) \\
& \frac{d C}{d T} H^{2 / 3}=F_{3 D}^{C}\left(\frac{T-T_{c}(H)}{(T H)^{2 / 3}}\right)
\end{aligned}
$$

where $F_{2 D}^{C}(x)$ and $F_{3 D}^{C}(x)$ are scaling functions.

On the other end the XY model can be considered a justified description for fields too weak to effectively break the XY symmetry. In this case the specific heat anomaly is expected to follow the scaling relation $16[17$.

$$
[C(H, T)-C(T, 0)] H^{\frac{\alpha}{2 \nu}}=G\left(\left(\frac{T}{T_{c}}-1\right) H^{\frac{-1}{2 \nu}}\right)
$$

where $G(x)$ is the scaling function and the parameters $\alpha=-0.007$ and $\nu=-0.669$ are the critical exponents characteristic for the 3D XY model.

Interestingly, although both the past and present analysis produce a similar of value $\mathrm{H}_{\mathrm{c} 2}$, specific heat data extended to 14 Tesla reveals that the high field data turns out to be not well described by the 3D Lowest Landau Level (3D-LLL) scaling.

A close look at Fig. 2 anveils that although the low field data might suggest an onset of convergence towards 3D-LLL scaling 6 , the additional higher field measurements indicate the 'non-convergence' continues. An attempt at describing the data using the 2D-LLL scaling (Fig $4 \mathrm{~b}$ ) is equally unsuccessful, although it seems to collapse the data slightly better. A representation of the data scaled within the 3D XY scaling ${ }^{16 \mid 18}$ framework (Figure 22) is equally unsatisfying. Suggesting that in fact none of the simple scaling scheme captures all the details of the specific heat anomaly. This is especially clear when comparing available datasets with nearperfect data collapse seen in $\mathrm{YBCO}^{1611920}$ or $\mathrm{BSCCO}^{21}$. Additionally comparing the specific heat anomaly of Nd$\mathrm{FeAs}(\mathrm{O}, \mathrm{F})^{11}$ and $\operatorname{SmFeAs}(\mathrm{O}, \mathrm{F})$ with their hydrogenated counterpart ${ }^{[13}$ reveals qualitative differences in the shape of the specific heat anomalies. This alone suggest that scaling approaches that proved useful in describing classic and cuprate superconductors are not sufficient to capture the details of the physics of the superconducting transition even within one family ('1111') of the pnictides.

\section{MAGNETORESISTANCE}

\section{Activated flux flow}

In order to perform electric transport measurements the crystals were removed from the calorimetric cell, glued to a silicon substrate and subsequently shaped
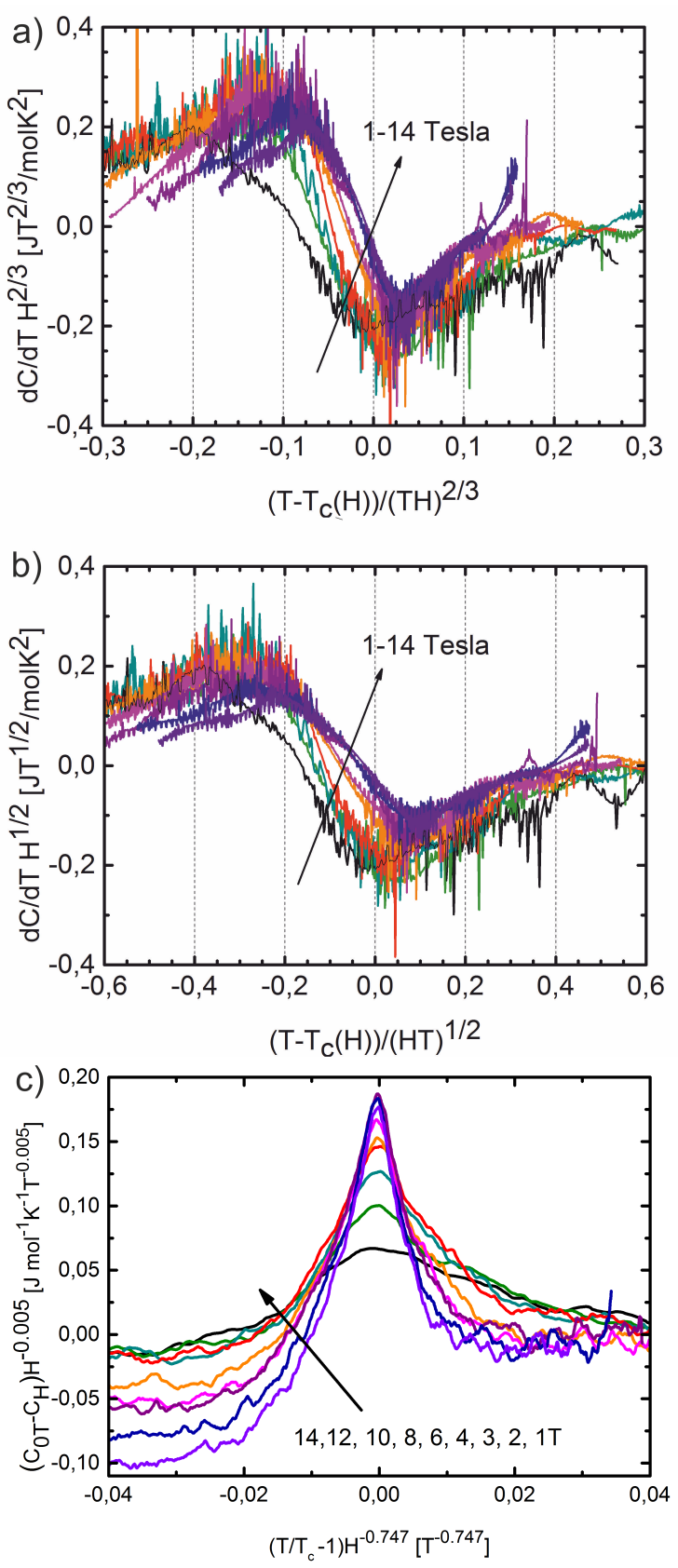

FIG. 2. Comparison of 2D (middle panel) and 3D (top panel) LLL-scaling schemes with the critical XY scaling (bottom panel). Best curve collapse for the LLL scaling was obtained for $T_{c}=50.2 \mathrm{~K}$ and $H_{c 2}=-3.1 \mathrm{~T} / \mathrm{K}$ and $T_{c}=49.7 \mathrm{~K}$ for XY scaling.

into a form of a Hall bar and contacted using the FIB technique ${ }^{9}$. The measurements were performed using a $1117.77 \mathrm{~Hz}$ excitation with peak current density of $20 \mathrm{~A} / \mathrm{cm}^{2}$ - well in the Ohmic regime ${ }^{22}$, in the same magnetic fields as the specific heat measurements. The resulting temperature and magnetic field dependence of the resistive transition is depicted in Fig 3. The most prominent feature is the previously reported broadening 


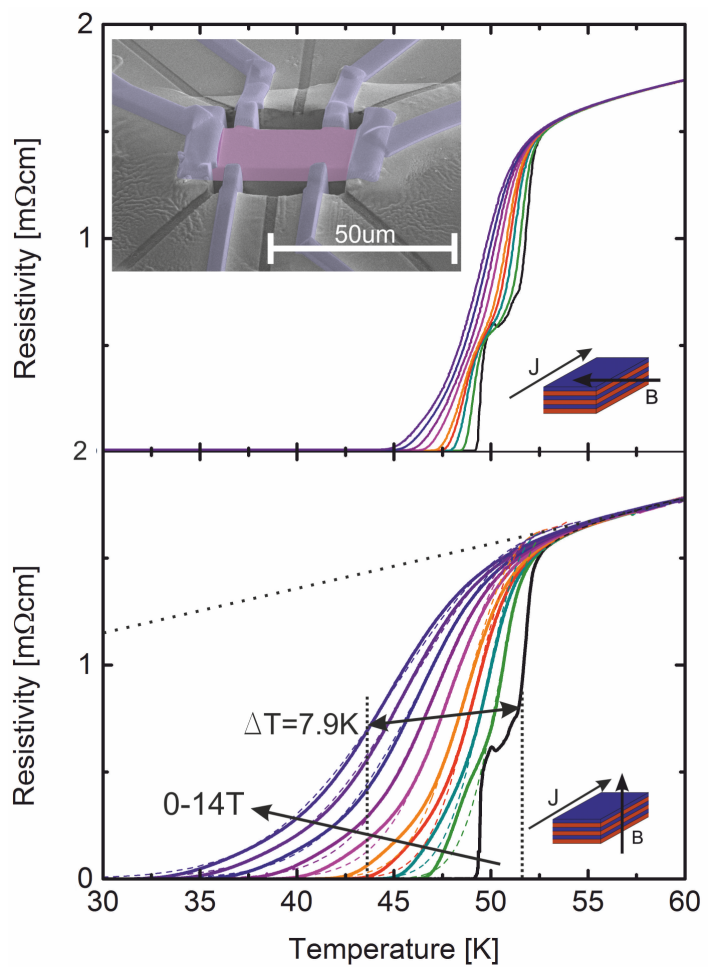

FIG. 3. The main panels demonstrate the temperature dependence of resistivity in magnetic fields along and perpendicular to the $c$-axis. The dashed lines in the lower panel are best fits to the activated flux flow model, see text. The inset shows an electron micrograph of the sample after preparation for transport measurements.

of the resistive transition with field applied along the $c$ axis.

It is worthwhile to investigate to what extent these $\rho(T, H)$ data can be used to extract the upper critical fields. So far there were two scenarios proposed in order to describe the shape of this transition: the first published study, on polycrystalline samples suggested that the transition width follows the 2D-LLL scaling relation for fields above 8 Tesl ${ }^{5}$. On the other hand later measurements on single crystal $\operatorname{SmFeAs}(\mathrm{O}, \mathrm{F})$ demonstrated the prevailing influence of vortex dynamic and activated flux flow as determining the broadening of the resistive transition 22 .

Indeed the basic model of activated flux motion as introduced by Tinkham ${ }^{23}$ parametrizes our data surprisingly well in high fields. For fields below 3 Tesla $(H c)$ there is some discrepancy at low temperature, originating from sample inhomogeneity. This feature becomes unimportant at high fields due to the intrinsic broadening of the transition and suppression of possible filamentary superconductivity.

$$
\frac{R}{R_{n}}=\left\{I_{0}\left[A(1-t)^{3 / 2} / 2 B\right]\right\}^{-2}
$$

Tinkhams model (eq. 1) describes the phenomenology of the resistive transition with only two material dependant parameters: $\mathrm{T}_{\mathrm{C}}$ and $A=C J_{c 0} / T_{c}$ where $C$ can be approximated as $C \approx \beta 8.07 \cdot 10^{-3} \mathrm{~T} \mathrm{~K}^{2} / A$, with $\beta$ being of the order of unity 23 .

In the case of $\operatorname{SmFeAs}(\mathrm{O}, \mathrm{F})$ we have obtained the best fits for $T_{c}=54 \mathrm{~K}$ and the value of $A(H)$ steadily increasing from $120 \mathrm{~T}$ and saturating at $380 \mathrm{~T}$ for fields above $4 \mathrm{~T}$. The outcome of the fitting procedure is represented by the dashed lines in the bottom panel of Figure 3. Our analysis displays three remarkable facts:

(1) The constant $A=\frac{U_{0}}{2 T}$ is proportional to the average vortex activation energy, thus its threefold increase could be thought of as a manifestation of field dependence of the pinning potential as suggested by Lee et al ${ }^{22}$ who found a transition between two regimes of $U_{0}(H)$ to occur at $3 \mathrm{~T}$. The saturated high field value of $A(H)$, yields $J_{c 0}=2.5 \cdot 10^{6} \mathrm{~A} / \mathrm{cm}^{2}$ very close to the value found for $\mathrm{YBCO} 23$.

(2) The value of $T_{c}$ for which the theory reproduces the data best is $\sim 54 \mathrm{~K}$ and remains almost the same for all magnetic fields. This remarkable fact was already noticed by Tinkham, originally attributed to the small depression of $T_{c}$ in magnetic field 23 . Within our framework the value of $54 \mathrm{~K}$ is significantly higher than the thermodynamic bulk transition temperature extracted from specific heat of the same crystal, the investigation of the specific heat data suggests a clear physical interpretation for the value of $T_{c}$ used in the activated flux flow model: it is the temperature defining the onset of superconducting fluctuations. Indeed in $\operatorname{SmFeAs}(\mathrm{O}, \mathrm{F})$ this temperature is about $54-55 \mathrm{~K}$ and seems to be not influenced by magnetic fields up to $14 \mathrm{~T}$. This opens a question to what degree the thermodynamic values of $H_{c 2}$ and $T_{c}$ are manifesting themselves in the phenomenology of the resistive transition.

\section{Superconducting fluctuation conductivity}

In the presence of flux motion the activated flux framework describes most of the shape of the resistive transition remarkably well. However the first $5-10 \%$ of the drop in resistance are usually dominated by the presence of superconducting fluctuations above the bulk transition temperature. To the best of our knowledge the superconducting fluctuation conductivity has been addressed twice for the '1111' family of superconductors5124. Pallecchi et al. recognized the shape of the resistive drop as following a 2D-LLL ${ }^{25 \mid 26}$ scaling relation. However later it was argued that such a result might have been the effect of using a polycrystalline sample ${ }^{6}$.

Similarly as in case of specific heat the LLL theory predicts that for sufficiently high fields the superconducting fluctuation contribution to conductivity is expected to follow specific dimension dependant scaling relations ${ }^{25 \mid 26}$. 


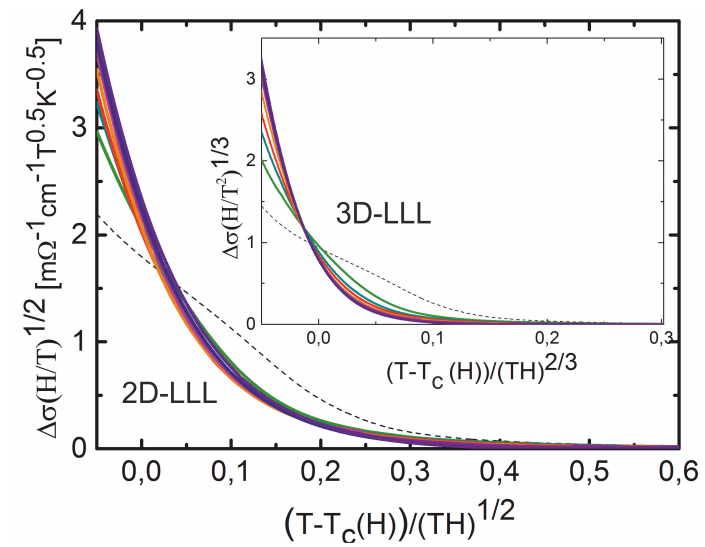

FIG. 4. Comparison of fluctuation conductivity data collapse in 2D (main panel) and 3D (inset) LLL scaling schemes for fields up to $14 \mathrm{~T}$. The dashed line represents the $1 \mathrm{~T}$ curve, for details see text. Best data collapse was obtained obtained for $\mathrm{T}_{\mathrm{c}}=50.2 \mathrm{~K}$ and $\mathrm{H}_{\mathrm{c} 2}=-3.1 \mathrm{~T} / \mathrm{K}$

$$
\begin{aligned}
& \triangle \sigma(H)=\left(\frac{T}{H}\right)^{\frac{1}{2}} F_{2 D}^{\sigma}\left(\frac{T-T_{c}(H)}{(T H)^{1 / 2}}\right) \\
& \triangle \sigma(H)=\left(\frac{T}{H}\right)^{\frac{1}{3}} F_{3 D}^{\sigma}\left(\frac{T-T_{c}(H)}{(T H)^{2 / 3}}\right)
\end{aligned}
$$

We have extracted the superconducting fluctuation contribution to conductivity by inverting the resistivity tensor and then subtracting the extrapolated normal state background. To investigate the dimensionality of these fluctuations we have plotted the conductivity data in scaled coordinates. As can be seen in Figure 4 the two 2D-LLL scaling collapses our data set far better then the $3 \mathrm{D}$ scheme: in the 2D case above $4 T$ (red curve) the collapse is nearly ideal whereas in the $3 \mathrm{D}$ case there is a considerable fanning out of the curves both below and above $T_{c}$. What is worth noting is that the best curve collapse was achieved with $T_{c}=50.2 \mathrm{~K}$ and $H_{c 2}=-3.1 \mathrm{~T} / \mathrm{K}$, very close the values obtained from the entropy conservation construction done on the raw specific heat data and from specific heat scaling. This comparison established the analysis of the superconducting fluctuation contribution to conductivity as a rather reliable method to establish $H_{c 2}$ and $T_{c}$

\section{SAMPLE QUALITY}

One of the primary concerns when discussing scaling of superconducting fluctuations is the availability of high quality crystals. In particular it is essential for this kind of studies to use single phase crystals, as inhomogeneous substitution throughout the sample could lead to the appearance of several superconducting transitions invalidating the scaling analysis. Proper care of this issue is especially important in materials known to be notoriously problematic to synthesize, such as the 1111 pnictides.

Indeed in the case at hand the 'two step' appearance of the resistive transition (Figure 33 could suggest a presence of a substantial inhomogeneity in the sample. It is instructive to compare this two step behaviour with the appearance of the specific heat anomaly. The specific heat data does not show any signatures of peak doubling which would be necessarily present for a two phase sample with two well defined transition temperatures. Additionally the inspection of magnetoresistance data shows that the first step in resistivity is suppressed by relatively weak fields suggesting that the two step appearance of the resistive transition can be attributed to filamentary/surface superconductivity and can be neglected at high fields where the LLL- fluctuations scaling should be applicable.

\section{CONCLUSIONS AND DISCUSSION}

In this study we have investigated the validity of both $\mathrm{XY}$ and LLL scaling schemes in application to a Sm(5) $\mathrm{FeAs}(\mathrm{O}, \mathrm{F})$, a representative of the pnictide superconductor family with the highest $T_{c}$. The analysis revealed that despite structural similarity to $\mathrm{YBCO}, \operatorname{SmFeAs}(\mathrm{O}$, $\mathrm{F}$ ) displays a range of behaviours that cannot be fully accounted for by theoretical approaches developed for the cuprates. This is particularly striking when considering specific heat, in YBCO both LLL and XY scaling are reasonably well describing experimental data and only considerable experimental effort settled the boundaries of applicability of both theories. For $\operatorname{SmFeAs}(\mathrm{O}, \mathrm{F})$ however none of the approaches provides convincing parametrization of experimental data. This is especially surprising in the case of LLL scaling. Considering the relatively low $T_{c}$ and its high Ginzburg number one would expect the LLL theory to be an adequate description of the condensate in the vicinity of $\mathrm{H}_{c 2}$ for relatively low field $\varsigma_{6|14| 15}$.

In this context the presence of $2 \mathrm{D}$ conductivity fluctuations is even more puzzling as recent studies of vortex transitions in $\operatorname{SmFeAs}(\mathrm{O}, \mathrm{F})^{10}$ conclusively showed that $\xi_{c}(T)$ remains larger than the inter-plane distance down to $T * \approx 0.8 T_{c}$ (Fig 5 questioning the $2 \mathrm{D}$ nature of superconducting fluctuations.

This apparent paradox might be explained by invoking the inherently multi-band nature of the pnictide superconductors $27-32$. In the case of weak inter-band interaction the main contribution to fluctuation conductivity would come from the band hosting the order parameter component with the largest $\xi_{a b}$, effectively 'shortening' all other conductivity fluctuations, inset Fig 5 . If these were of $2 \mathrm{D}$ character one could indeed expect the 2D-LLL to parametrize conductivity well for sufficiently high magnetic fields, Fig 5. On the other hand specific heat measures the total change of entropy, and thus would pick up contributions from both 2D and 3D fluctuations leading to the breakdown of simple LLL-scaling. 


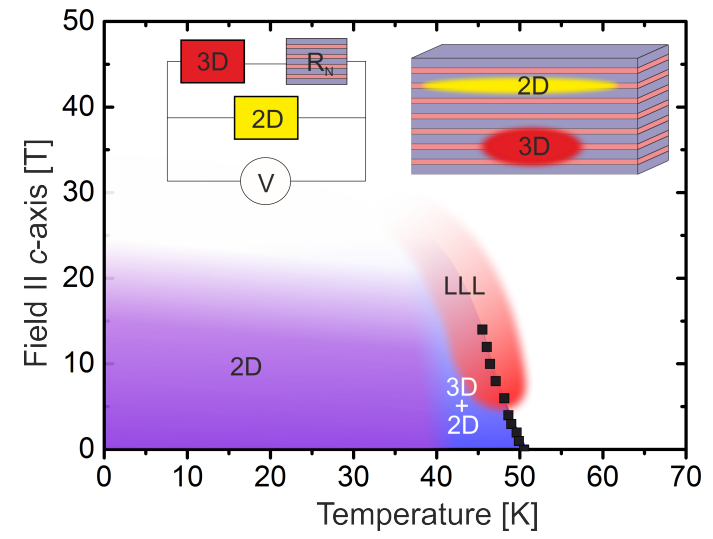

FIG. 5. A schematic 'Phase diagram' of $\operatorname{SmFeAs}(\mathrm{O}, \mathrm{F})$. The insets shows how 2D fluctuations extended in the $a b$-plane could 'screen' existing 3D fluctuations and lead to the observation of $2 \mathrm{D}-\mathrm{LLL}$ scaling.
In the case of $\operatorname{SmFeAs}(\mathrm{O}, \mathrm{F})$ it was shown that two gaps open at the same temperature with $\Delta_{1}(0)=18 \mathrm{meV}$ and $\Delta_{2}(0)=6.2 \mathrm{meV}$. Taking into account the very $2 \mathrm{D}$ character of its Fermi surface, the standard BCS expression for the coherence length $\xi_{0}=\frac{\hbar v_{F}}{\pi \Delta}$ suggests that at least one component of the order parameter could indeed be highly two dimensional.

In this picture at temperatures above $T_{c}$ the properties of $\operatorname{SmFeAs}(\mathrm{O}, \mathrm{F})$ are determined by a combination of $2 \mathrm{D}$ and $3 \mathrm{D}$ fluctuations. On cooling below bulk $T_{c}$ the 3D component begins to dominate most of the phenomenology until $\xi_{c}(T)$ becomes shorter than the distance between adjacent FeAs layers at which point the dominating component of the order parameter becomes $2 \mathrm{D}$.

\section{ACKNOWLEDGMENTS}

We would like to thank A. Zheludev, K. Povarov and V. B. Geshkenbein for many enlightening discussions that helped improve this manuscript.
* Corresponding author: galeskis@phys.ethz.ch

1 Y. Kamihara, T. Watanabe, M. Hirano, and H. Hosono, Journal of the American Chemical Society 130, 3296 (2008)

2 J. Paglione and R. L. Greene, Nature Physics 6, 645 (2010),

3 N. D. Zhigadlo, S. Katrych, Z. Bukowski, S. Weyeneth, R. Puzniak, and J. Karpinski, Journal of Physics: Condensed Matter 20, 342202 (2008).

${ }^{4}$ H.-S. Lee, M. Bartkowiak, J.-H. Park, J.-Y. Lee, J.-Y. Kim, N.-H. Sung, B. Cho, C.-U. Jung, J. Kim, and H.-J. Lee, Physical Review B 80, 144512 (2009)

5 I. Pallecchi, C. Fanciulli, M. Tropeano, A. Palenzona, M. Ferretti, A. Malagoli, A. Martinelli, I. Sheikin, M. Putti, and C. Ferdeghini, Physical Review B 79, 104515 (2009)

${ }^{\circ}$ U. Welp, C. Chaparro, A. E. Koshelev, W. K. Kwok, A. Rydh, N. D. Zhigadlo, J. Karpinski, and S. Weyeneth, Physical Review B 83, 100513 (2011).

7 S. van Herwaarden, "Gas Nanocalorimeters XEN-39390 series technical note." (2016).

${ }^{8}$ S. Galeski, K. Mattenberger, and B. Batlogg, Physical Review B 94, 140402 (2016).

${ }^{9}$ P. J. W. Moll, R. Puzniak, F. Balakirev, K. Rogacki, J. Karpinski, N. D. Zhigadlo, and B. Batlogg, Nature materials 9, 628 (2010).

${ }^{10}$ P. J. W. Moll, L. Balicas, V. Geshkenbein, G. Blatter, J. Karpinski, N. D. Zhigadlo, and B. Batlogg, Nature Materials 12, 134 (2012).

${ }^{11}$ U. Welp, R. Xie, A. E. Koshelev, W. K. Kwok, P. Cheng, L. Fang, and H.-H. Wen, Physical Review B 78, 140510 (2008)

${ }^{12}$ U. Welp, R. Xie, A. E. Koshelev, W. K. Kwok, H. Q. Luo, Z. S. Wang, G. Mu, and H. H. Wen, Physical Review B 79, 094505 (2009)

13 N. D. Zhigadlo, "Unpublished," (2015).
14 Z. Tesanovic and A. V. Andreev, Physical Review B 49, 4064 (1994),

is S. W. Pierson, T. M. Katona, Z. Tes, and O. T. Valls, Physical Review B 53, 8638 (1996).

16 O. Jeandupeux, A. Schilling, H. R. Ott, and A. Van Otterlo, Physical Review B 53, 12475 (1996).

17 M. B. Salamon, J. Shi, N. Overend, and M. A. Howson, Physical Review B 47, 5520 (1993)

18 I. D. Lawrie, Physical Review B 50, 9456 (1994)

19 U. Welp, S. Fleshier, W. K. Kwok, R. A. Klemm, V. M. Vinokur, J. Downey, B. Veal, and G. W. Crabtree, Physical Review Letters 67, 22 (1991).

20 N. Overend, M. A. Howson, I. D. Lawrie, S. Abell, P. J. Hirst, C. Chen, S. Chowdhury, J. W. Hodby, S. E. Inderhees, and M. B. Salamon, Physical Review B 54, 9499 (1996)

${ }^{21}$ N. Kobayashi, K. Egawa, K. Miyoshi, H. Iwasaki, H. Ikeda, and R. Yoshizaki, Physica C: Superconductivity 219, 265 (1994)

${ }^{22}$ H.-S. Lee, M. Bartkowiak, J. S. Kim, and H.-J. Lee, Physical Review B 82, 104523 (2010).

${ }_{23}$ M. Tinkham, Physical Review Letters 61, 1658 (1988)

24 S. L. Liu, W. Haiyun, and B. Gang, Physics Letters A 374, 3529 (2010).

25 S. Ullah and A. T. Dorsey, Physical Review Letters 65, 2066 (1990).

${ }_{26}$ S. Ullah and A. T. Dorsey, Physical Review B 44, 262 (1991)

27 S. Kawasaki, K. Shimada, G. F. Chen, J. L. Luo, N. L. Wang, and G.-Q. Zheng, Physical Review B 78, 220506 (2008)

${ }_{28}$ P. Szabo, Z. Pribulova, G. Pristas, S. L. Budko, P. C. Canfield, and P. Samuely, Physical Review B 79, 012503 (2009)

${ }^{29}$ V. Stanev, J. Kang, and Z. Tesanovic, Physical Review B 
78, 184509 (2008)

so F. Hunte, J. Jaroszynski, A. Gurevich, D. C. Larbalestier, R. Jin, A. S. Sefat, M. A. Mcguire, B. C. Sales, D. K. Christen, and D. Mandrus, Nature Letters 453, 903 (2008).

31 R. S. Gonnelli, D. Daghero, M. Tortello, G. A. Ummarino, V. A. Stepanov, J. S. Kim, and R. K. Kremer, Physical
Review B 79, 184526 (2009)

32 D. Daghero, M. Tortello, R. S. Gonnelli, V. A. Stepanov, N. D. Zhigadlo, and J. Karpinski, Physical Review B 80, 060502 (2009). 